\title{
Society for Pediatric Research Presidential Address 1990: Pediatric Research-Integrated Evaluations to Achieve Insights into Organ Function
}

\author{
ALAN H. JOBE
}

Harbor-UCLA Medical Center, Department of Pediatrics, Torrance, California 90509

\begin{abstract}
Members of the Society and guests, it is a pleasure and honor to address you this morning as a spokesperson for pediatric research. In thinking about this task, I sought opinions as to content from a number of my mentors and colleagues. About half recommended that I talk about my science and the other half felt that this address is a unique opportunity to focus on a controversy that impacts pediatric research. I will try to do a bit of both, in that I will use several examples of research from my laboratory to make the central point of this talk-while the reductionist approach to research is both compelling and trendy, it is only useful when interpreted within the context of integrated organ system and body function.
\end{abstract}

As pediatricians, our interests are directed toward strategies for improving the lot of the world's children. As research clinicians in a modern western society, most of our research questions, even the most profound and mechanistic, are egocentric and culturally biased in the extreme. Deaths in children in the developing world exceed those in developed countries by some 70 -fold, and most of those deaths result from preventable causes such as diarrhea, measles, malaria, and tetanus. The major barriers to improvements in global childhood health are social and political rather than medical. In our own country, the progress in reducing infant mortality has halted and almost certainly will reverse. Immunization levels among our children are decreasing as access to health care is reduced. Although I will not dwell on the depressing statistics of international health, I feel strongly that we all need to keep in mind the limited impact of recent research progress on molecular mechanisms of pediatric diseases within the context of global health or even national health statistics. These opening comments are only meant to assure some perspective and humility about the role of pediatric research within pediatrics in general.

I find an uncomfortable tension within pediatric departments concerning the type of research that is viewed as good versus mundane. There is a clearly perceived hierarchy of research quality based upon the categorization of that research. Good research is viewed as basic, mechanistic, hypothesis-driven investigative work that at the overt or covert level is assumed to be bench research, at the most molecular level possible. In contrast, bad, mundane, or-most repugnant-unfundable research, is likely to be classified as applied, descriptive, physiologic, or phenomenologic. The view that basic research is molecular is incorrect-basic research, according to Lewis Thomas (1), is distinguished from applied research by the single distinction of uncertainty. Basic research asks questions about the unknown, be that a molecular mechanism or an epidemiologic theory. Therefore, by definition, most of pediatric research would fall into the category of applied research, inasmuch as the focus is

Supported by grants HD-1 1932, HD-12714, and HD-20618 from NIH-Child Health Development. the improvement in child health either for the individual or for children as a group. Basic research is not necessarily cloning the gene for a rare complement defect, because the techniques for cloning are now routine. Such a research project is applied molecular research just as the development of a vaccine is applied research once the offending organism has been isolated and its biology elucidated. The strength of the basic scientist is in the development of research concepts and tools. The strength of the pediatric investigator should be in the application of those tools to important pediatric problems because of his or her unique clinical perspective.

Mechanistic also is a catch word that gives a research project an aura of quality and profundity. The difficulty with this categorization is that one person's mechanism is another person's description of the system in question (2). The ultimate description is the sequence of a gene. There is a clear hierarchy in the process of asking questions in a clinical specialty that begins with clinical observation, which is often pejoratively referred to as phenomenology. However, phenomenology is the substance of clinically relevant questions. The social pecking order of the "hierarchy of prestige" (3) and rewards for research then is clinical observation, physiology, cell biology, and, finally, molecular biology. This view is stated rather succinctly by Francis Crick (4), who wrote, "Almost all aspects of life are engineered at the molecular level ... All approaches at a higher level are suspect until confirmed at the molecular level." Although this statement is ultimately true, I am quite certain that the converse is equally true. All molecular observations must be integrated into the functioning organism to be correctly interpreted.

I will use several examples from my laboratory to illustrate how misleading reductionist approaches can be when applied to complicated biologic systems. After Ph.D. training at the Salk Institute, where I worked on regulation of the lac operon in $E$. coli, I chose fellowship training with Louis Gluck. My research goal in 1975 was to understand respiratory distress syndrome at the quantitative level by applying mass action concepts that I had learned studying the lac operon to respiratory distress syndrome. That remains my research focus now, some 15 years later. My research is almost entirely whole-animal based, inasmuch as an integrated appreciation of lung function in the preterm demands this approach-a strategy diametrically opposite to the molecular investigations of my thesis. The use of animal models was driven by the question rather than by my preference for the complexities of such experimental strategies.

Although the question of maternal corticosteroid treatment and fetal lung maturation may seem a bit dated, I am convinced that the general strategy to mature the fetus at risk for preterm delivery can be better exploited to optimize the outcome for preterm infants. Neonatologists have focused on the lung to the detriment of understanding the general maturational events of other organ systems in the fetus. Liggins (5) initially observed in 
1969 that corticosteroids caused preterm labor in sheep and that some preterm lambs had mature lungs. He suggested that corticosteroids induced surfactant synthesis. Subsequently, many investigators demonstrated induced lung maturation with corticosteroids (6). In vitro studies using organ explants and isolated cells clearly documented increased surfactant synthesis. Although other effects of corticosteroids on the fetal lung such as stimulated FFA synthesis (7) and structural maturation (8) were documented, the consensus understanding is that corticosteroid-induced lung maturational responses predominantly result from increased surfactant synthesis (9).

We felt that studies of fetal lungs did not adequately test the synthesis hypothesis because neonatal adaptation to air breathing is such a complex process. Therefore, we have evaluated integrated lung function after fetal corticosteroid treatments in rabbits and sheep. We anticipated that the corticosteroids would increase surfactant pools slightly and wanted to eliminate that variable by using surfactant treatment to overcome any differences in surfactant pools. In initial experiments in preterm rabbits, we found that maternal corticosteroid treatment had little effect on dynamic lung compliance (10). The unanticipated result was a striking synergistic effect of the maternal corticosteroid treatments and surfactant on lung compliance. In contrast, corticosteroid treatments of fetal lambs did improve postnatal lung compliances, but striking synergistic effects with surfactant were again noted (11). Improved surfactant responses after maternal corticosteroid treatments also have been noted in the clinical trials (12).

When we measured the amount of surfactant as saturated phosphatidylcholine in the airspaces of the preterm ventilated rabbits and sheep, we found no increase in surfactant in the corticosteroid-treated groups $(11,13)$. Maternal corticosteroid treatments did not increase alveolar surfactant pools at any gestation tested in rabbits (13). This result differs from other observations in the literature, but is quite consistent with recent reports from Fiasconi et al. (14) and Liggins et al. (15). The inconsistency results in part from measurements made with fetal rather than neonatal lungs.

We have been interested for many years in the tendency of the preterm ventilated lung to develop pulmonary edema. We asked if a maturational event might be a decreased leak of vascular proteins into the lungs of corticosteroid-treated preterm animals and found that fetal corticosteroid exposure resulted in a $50 \%$ reduction of the vascular-to-alveolar leak of radiolabeled albumin in preterm rabbits and a 4-fold reduction in this protein leak in preterm ventilated lambs $(10,11,13)$. These striking effects probably are clinically important, inasmuch as proteinaceous pulmonary edema inactivates surfactant and no doubt contributes to the respiratory failure in respiratory distress syndrome (16).

Another interesting observation that may explain the decreased incidence of respiratory distress syndrome in fetuses exposed to corticosteroids is the shift in the surfactant-compliance doseresponse curves in corticosteroid-treated preterm rabbits $(17,18)$. Corticosteroid treatments decreased the minimal pool sizes needed to get compliance responses and increased the maximal responses achieved for both endogenous surfactant pool sizes and treatment doses of surfactant. When interpreted within the clinical context, a reasonable explanation for the decreased incidence of respiratory distress syndrome in the human is that corticosteroids simply alter the dose-response curve and thus prevent respiratory distress syndrome in some infants without altering surfactant pool sizes.

These observations of the effects of corticosteroids on preterm lung function serve to illustrate two points: the responses of a developing organ to an inducing agent can be very complex, and the responses may become apparent only with an integrated functional evaluation of that organ. In these rabbit and lamb studies, surfactant pool sizes were not altered by corticosteroid treatments, in direct contrast to the predictions from the cell biology. Rather, multiple other functional and structural changes in the lung altered lung performance in ways that mimicked those that would be anticipated if surfactant pool sizes had increased. The reductionist concept that corticosteroids bind to a receptor that signals increased surfactant synthesis is simplistic in the extreme and perhaps not even correct in the preterm infant at a point in gestation when variability is possible. Rather, corticosteroids induced global fetal maturation of multiple organ systems. There is compelling information that such treatments impact gut, skin, and kidney maturation, as well as lung maturation. In contrast to other notable therapeutic attempts in neonatology, in the case of corticosteroids, we are using the right therapy for preterm infants but for the wrong reasons.

My next example of the complexity of the surfactant system focuses on the fluxes of surfactant phosphatidylcholine within the lung. These studies were started to approach the goal of understanding respiratory distress syndrome at the quantitative level. In my initial experiments during my fellowship, I asked the descriptive question, "What were the kinetics of surfactant secretion in vivo?" Using preterm, newborn, and adult rabbit models, I found that after the injection of labeled precursors, the packaging and subsequent secretion of surfactant phosphatidylcholine was not a rapid process (19). There were delays between synthesis and the initiation of secretion as well as very long periods required to achieve maximal secretion. For example, maximal labeling of alveolar surfactant was not measured until about $30 \mathrm{~h}$ in newborn rabbits and $40 \mathrm{~h}$ in newborn lambs after radiolabeled precursor injection $(20,21)$. These early experiments and subsequent measurements of incorporation rates in animals at different gestational ages can be summarized as the first principle of the surfactant system: neither de novo synthesis nor secretion of de novo synthesized surfactant are rapidly responsive to stresses such as preterm birth. Stated another way, short-term regulation of surfactant phosphatidylcholine pools does not primarily involve de novo synthesis pathways. Shortterm adjustments in surfactant pools are accomplished by the release of tissue stores to the airspaces, a process that is under many controls that include at least $\beta$-agonists and lung stretch (6). Surfactant deficiency in the preterm infant is almost certainly not the inability to synthesize and secrete surfactant, but rather inadequate stores, a point that becomes important to the concept of surfactant treatments.

My initial experiments were directed toward the anabolic side of the mass action equation, but, in retrospect, most of the interesting differences between the developing and adult lung result from differences in catabolism. In experiments conducted primarily by Harris Jacobs when he was a fellow in my laboratory, we tried to quantify the relationships between the intracellular storage pool, the lamellar bodies, and the alveolar surfactant $(20,22,23)$. We used different radiolabeled precursors of phosphatidylcholine to track the fate of both de novo synthesized surfactant phosphatidylcholine and alveolar surfactant phosphatidylcholine. The striking results were that, when expressed on a per $\mathrm{kg}$ body weight basis, de novo synthesis was less in the newborn than in the adult rabbit although tissue storage pools, secretory fluxes, and alveolar pools were much larger. The explanation for these differences was that the developing lung recycled surfactant phosphatidylcholine very efficiently, whereas the adult lung catabolized the surfactant phosphatidylcholine. This observation can be extended to the preterm infant with surfactant deficiency who then received surfactant treatments. Preterm lambs at $32 \mathrm{~d}$ gestational age have severe respiratory failure and surfactant deficiency resulting in death by about $5 \mathrm{~h}$ despite mechanical ventilation (24). However, if these animals were treated with a single dose of surfactant at birth, they could be supported easily with quite good lung function for at least $24 \mathrm{~h}$. The striking observation was that although the surfactant phosphatidylcholine was lost from the airspaces over $24 \mathrm{~h}$, there was very little catabolic activity in the lungs. These measurements, together with labeling patterns in lamellar bodies, implied that 
the surfactant phosphatidylcholine used for treatment "charged" the endogenous surfactant metabolic pathways with substrate, which then was recycled with maintenance of surfactant function (25). These experiments illustrate a second principle of surfactant metabolism: the primary difference between the developing and adult lung is the lack of catabolism in the developing lung.

An understanding of surfactant function in respiratory distress syndrome is complicated by many other factors besides surfactant pools and fluxes. In our earliest surfactant treatment studies using very premature lambs, we noted that the surfactant treatment responses were not sustained for a long period of time (26). Our initial assumption was that the surfactant was lost from the lung, but, although this was in part true, we found that the surfactant had lost all of its desirable surface properties when studied in vitro. Samples from the airspaces of infants with respiratory distress syndrome were used to demonstrate that the minimal surface tensions were very abnormal as compared with controls (16). However, separation of the surfactant from the soluble proteins by a simple centrifugation step resulted in isolation of surfactant with very good surface properties. We found that the addition of the soluble proteins from the airspaces of preterm animals or infants to surfactant will reversibly inactivate the surfactant, whereas proteins from control infants were less inhibitory. This demonstration of surfactant inactivation is just one example of the difficulty of interpreting surfactant function and metabolism in vivo because of multiple phenomena that occur within the microenvironment of the alveolus. A third principle of the surfactant system is that surfactant function cannot be directly related to surfactant quantity, especially in the injury-prone preterm lung.

I have briefly reviewed several studies from my laboratory to simply point out that understanding the complexity of systems such as surfactant or diseases such as respiratory distress syndrome requires a systems approach to the integrated metabolism and physiology. The experiments that we are now doing certainly are stimulated by the results of my colleagues working on the cell biology and molecular biology of the lung and the surfactant system. The molecular work has and will continue to provide the tools for the in vivo experiments. The point is that progress is made by asking questions in a hierarchic order-the phenomenon stimulates the descriptive work, which in turn should motivate a search for molecular and cell biologic explanations. These explanations at the molecular level then need to be verified, calibrated as to their importance, and integrated into an understanding of the phenomenon. Each of us has a role to play at the different levels. As long as the question is approached analytically and imaginatively, one level of the hierarchy should not carry a value higher or lower than any other level. This thought was clearly stated by William Silverman (3) in his editorial in Pediatric Research titled "The divisive effect of reductionist snobbery":

The reductionist approach to complexity in medicine is a powerful strategy, but it must not be regarded as the be-all and end-all of research. The study of intracellular phenomena and the study of whole body events (singly and in collections) should not be undertaken in isolation from one another. And they must be equally valued and equally disciplined if the wide gap between miracles promised and miracles delivered is to be narrowed in medicine.

Acknowledgements. The author thanks Machiko Ikegami, M.D., Ph.D., who has been his research colleague for many years, and the neonatal fellows who have worked in his laboratory for their help.

\section{REFERENCES}

1. Thomas L 1978 Hubris in science? Science 200:1459-1462

2. Thurlbeck WM 1989 Perestroika, fashion, and the universal glue. Am Rev Respir Dis 139:1280-1281

3. Silverman WA 1983 The divisive effect of reductionist snobbery. Pediatr Res 17.518

4. Crick F 1988 What Mad Pursuit: A Personal View of Scientific Discovery. Basic Books, New York

5. Liggins GC 1969 Premature delivery of foetal lambs infused with glucocorticoids. J Endocrinol 45:515-523

6. Rooney SA 1985 The surfactant system and lung phospholipid biochemistry. Am Rev Respir Dis 131:439-460

7. Maniscalco WM, Finkelstein JN, Parkhurst A 1985 Dexamethasone increased de novo fatty acid synthesis in fetal rabbit lung explants. Pediatr Res 12:1272 1277

8. Kauffman SL 1977 Acceleration of canalicular development in lungs of fetal mice exposed transplacentally to dexamethasone. Lab Invest 36:395-401

9. Ballard PL 1986 Hormones and Lung Maturation. Springer-Verläg, Berlin

10. Ikegami M, Berry D, Elkday T, Pettenazo A, Seidner S, Jobe A 1987 Corticosteroids and surfactant change lung function and protein leaks in the lungs of ventilated premature rabbits. J Clin Invest 79:1371-1378

11. Ikegami M, Polk D, Lewis J, Tabor B, Yamada T, Jobe A 1990 Fetal corticosteroid and thyroid releasing hormone effects on lung function following delivery and ventilation of preterm lambs. Am Rev Respir Dis 141:A341(abstr)

12. Farrell EE, Silver RK, Kimberlin LV, Wolf ES, Dusik JM 1989 Impact of antenatal dexamethasone administration on respiratory distress syndrome in surfactant-treated infants. Obstet Gynecol 161:628-633

13. Ikegami M, Jobe A, Seidner S, Yamada T 1989 Gestational effects of corticosteroids and surfactant in ventilated rabbits. Pediatr Res 25:32-37

14. Fiascone JM, Jacobs HC, Fernando R, Moya FR, Mercurio MR, Lima DM 1987 Betamethasone increases pulmonary compliance in part by surfactantindependent mechanisms in preterm rabbits. Pediatr Res 22:730-735

15. Liggins GC, Schellenberg J, Manzai M, Kitterman J, Lee CH 1988 Synergism of cortisol and thyrotropin-releasing hormone in lung maturation in fetal sheep. J Appl Physiol 65:1880-1884

16. Ikegami M, Jacobs H, Jobe A 1983 Surfactant function in respiratory distres syndrome. J Pediatr 102:443-447

17. Ikegami M, Jobe A, Yamada T, Seidner S 1989 Relationship between alveola saturated phosphatidylcholine pool sizes and compliance of preterm rabbit lungs. Am Rev Respir Dir 139:367-369

18. Seidner S, Pettenazzo A, Ikegami M, Jobe A 1988 Corticosteroid potentiation of surfactant dose response in preterm rabbits. $J$ Appl Physiol 64:2366-237

19. Jobe A Kirkpatrick E, Gluck L 1978 Lecithin appearance and apparent biologic half-life in term newborn rabbit lung. Pediatr Res 12:669-675

20. Jacobs H, Jobe A, Ikegami M, Jones S 1982 Surfactant phosphatidylcholine source, fluxes and turnover times in 3-day-old, 10-day-old and adult rabbits. J Biol Chem 257:1805-1810

21. Ikegami M Jobe A, Nathanielsz PW 1981 The labeling of pulmonary surfactant phosphatidylcholine in newborn and adult sheep. Exp Lung Res 2:197-206

22. Jacobs H, Jobe A, Ikegami M, Conaway D 1983 The significance of reutilization of surfactant phosphatidylcholine. J Biol Chem 258:4156-4165

23. Jacobs HC, Ikegami M, Jobe AH, Berry DD, Jones S 1986 Reutilization of surfactant phosphatidylcholine in adult rabbits. Biochim Biophys Acta 837:77-84

24. Ikegami M, Jobe A, Yamada T, Priestly A, Ruffini L, Rider E, Seidner S 1989 Surfactant metabolism in surfactant-treated preterm ventilated lambs. J App Physiol 67:429-437

25. Jobe A, Ikegami M, Seidner S, Pettenazzo A, Ruffini L 1989 Surfactant phosphatidylcholine metabolism and surfactant function in preterm, ventilated lambs. Am Rev Respir Dis 139:352-359

26. Ikegami M, Jobe AH, Glatz T 1981 Surface activity following natural surfactan treatment in premature lambs. J Appl Physiol 51:306-312 